\title{
Analysis of Bovine Viral Diarrhea Viruses-infected monocytes: identification of cytopathic and non- cytopathic biotype differences
}

Mais Ammari ${ }^{1 \dagger}$, Fiona M McCarthy ${ }^{1,2 \dagger}$, Bindu Nanduri ${ }^{1,2}$, Lesya M Pinchuk ${ }^{1 *}$

From Seventh Annual MCBIOS Conference. Bioinformatics: Systems, Biology, Informatics and Computation Jonesboro, AR, USA. 19-20 February 2010

\begin{abstract}
Background: Bovine Viral Diarrhea Virus (BVDV) infection is widespread in cattle worldwide, causing important economic losses. Pathogenesis of the disease caused by BVDV is complex, as each BVDV strain has two biotypes: non-cytopathic (ncp) and cytopathic (cp). BVDV can cause a persistent latent infection and immune suppression if animals are infected with an ncp biotype during early gestation, followed by a subsequent infection of the cp biotype. The molecular mechanisms that underscore the complex disease etiology leading to immune suppression in cattle caused by BVDV are not well understood.
\end{abstract}

Results: Using proteomics, we evaluated the effect of $\mathrm{cp}$ and ncp BVDV infection of bovine monocytes to determine their role in viral immune suppression and uncontrolled inflammation. Proteins were isolated by differential detergent fractionation and identified by 2D-LC ESI MS/MS. We identified 137 and 228 significantly altered bovine proteins due to ncp and cp BVDV infection, respectively. Functional analysis of these proteins using the Gene Ontology (GO) showed multiple under- and over- represented GO functions in molecular function, biological process and cellular component between the two BVDV biotypes. Analysis of the top immunological pathways affected by BVDV infection revealed that pathways representing macropinocytosis signalling, virus entry via endocytic pathway, integrin signalling and primary immunodeficiency signalling were identified only in ncp BVDV-infected monocytes. In contrast, pathways like actin cytoskeleton signalling, RhoA signalling, clathrinmediated endocytosis signalling and interferon signalling were identified only in $\mathrm{cp}$ BDVD-infected cells. Of the six common pathways involved in cp and ncp BVDV infection, acute phase response signalling was the most significant for both BVDV biotypes. Although, most shared altered host proteins between both BVDV biotypes showed the same type of change, integrin alpha 2b (ITGA2B) and integrin beta 3 (ITGB3) were down- regulated by ncp BVDV and up- regulated by cp BVDV infection.

Conclusions: This study shows that, as we expected, there are significant functional differences in the host proteins that respond to cp or ncp BVDV infection. The combined use of GO and systems biology network modelling facilitated a better understanding of host-pathogen interactions.

\footnotetext{
* Correspondence: pinchuk@crm.msstate.edu

+ Contributed equally

'Department of Basic Sciences, Mississippi State University, Mississippi State,

MS 39762, USA

Full list of author information is available at the end of the article
} 


\section{Background}

Bovine Viral Diarrhea Virus (BVDV) infections are seen in all ages and breeds of cattle worldwide and have significant economic impact due to productive and reproductive losses [1,2]. BVDV is a single-stranded, positive-sense RNA virus, belonging to the Flaviviridae family, genus Pestivirus [1,2]. BVDV genotypes are classified according to their effects in cell cultures into two different biotypes: non-cytopathic (ncp) and cytopathic (cp). Different isolates of both forms commonly exhibit antigenic differences [3,4]. The pathogenesis of the disease caused by BVDV is complex and involves persistent infection (PI) and immune suppression with the ncp biotype during early gestation, followed by an acute infection by a cp biotype $[5,6]$. PI animals shed virus and initiate further virus replication and genetic variation [5,6]. The fatal form of BVDV mucosal disease only occurs in animals carrying the ncp biotype and become exposed to the cp biotype [6]. Even though BVDV is one of the most studied infective agents in cattle it is probably one of the least understood. This is mainly because BVDV are a group of multiple viruses affecting virtually all organs and system in the body, including innate and adaptive immune system [7]. Identifying the molecular mechanisms and developing strategies for controlling the spread of the virus are the challenges faced by BVDV researchers.

Taking into consideration that PI animals are the major disseminators of BVDV in the cattle population, we hypothesized that low doses of BVDV infection can provide some answers in the BVDV pathogenesis. In our earlier work we assessed selective and non-selective antigen uptake mechanisms in BVDV-infected monocytes and outlined some similarities and differences between the two BVDV biotypes [8]. Following the differences in the antigen uptake function of monocytes and using the same infection protocols we determined the TLR, cytokine and costimulatory molecules gene expression in the infected cells [9]. Francini et al. using high doses of BVDV in vitro did not detect significant differences in the TLR expression levels in bovine macrophages [10]. Using a proteomic approach, we demonstrated that cp BVDV biotype affected the expression of proteins related to professional antigen presentation. In particular, proteins related to immune responses, such as cell adhesion, apoptosis, antigen uptake, processing and presentation, acute phase response proteins, MHC class I- and class II-related proteins and other molecules involved in immune function of professional antigen presentation have been significantly altered after BVDV infection [9]. Finally, we demonstrated the differential effects of $\mathrm{cp}$ and ncp BVDV biotypes on the expression levels of the protein kinases and related proteins affecting the development of infection and antiviral mechanisms in bovine monocytes [11].

To better understand the complexity of the mechanisms by which the cp and ncp BVDV cause disease, and to identify biotype-related differences in significant biological functions and pathways here we further analyzed the expression of immunologically important proteins by combined use of GO and systems biology network modelling.

\section{Results}

Our overall approach to determine the differential effects of cp and ncp BVDV infection on the monocytedependent innate and adaptive immune responses involved identification of differentially expressed proteins in each type of infection followed by functional modelling using both GO and Ingenuity Pathway Analysis (IPA) pathway and network analysis (Figure 1). The results of each of these steps are presented in more detail in the following sections.

\section{Protein identification and differentially expressed proteins in ncp and cp BVDV-infected monocytes}

We initially identified a total of 2489, 2356 and 2028 bovine proteins from uninfected, ncp and cp BVDVinfected bovine monocytes, respectively. By comparing ncp BVDV-infected host proteins to their uninfected counterparts we were able to determine up- and downregulated host proteins occurring in either $\mathrm{cp}$ or ncp BVDV infection (Figure 2). This gave us a total of 1137 (31.4\%) altered proteins unique to ncp BVDV-infected monocytes and 929 (27.0\%) altered proteins unique to cp BVDV-infected cells.

Compared to uninfected monocytes, ncp BVDV altered the expression of 137 host proteins with 55 $(40.2 \%)$ being down-regulated and $82(59.8 \%)$ being upregulated (Figure 3, additional file 1). In comparison, cp BVDV altered the expression of 228 host proteins of which 164 (72.0\%) were down-regulated and 64 (28.0\%) were up-regulated, compared to uninfected monocytes (Figure 3, additional file 2). Of these differentially expressed proteins, 69 host proteins were common to ncp and cp BVDV infections. The expression trends for these shared proteins were similar for all except for integrin alpha $2 \mathrm{~b}$ (ITGA2B) and integrin beta 3 (ITGB3), that were down- regulated by ncp BVDV and up- regulated by cp BVDV infection.

Comparison of proteins unique to ncp BVDV-infected monocytes (1137) with proteins unique to cp BVDVinfected cells (929) showed that 240 (13.2\%) common host response proteins, 897 (49.1\%) and 689 (37.7\%) proteins unique to ncp and cp BDVD-infected monocytes, respectively (data not shown). 


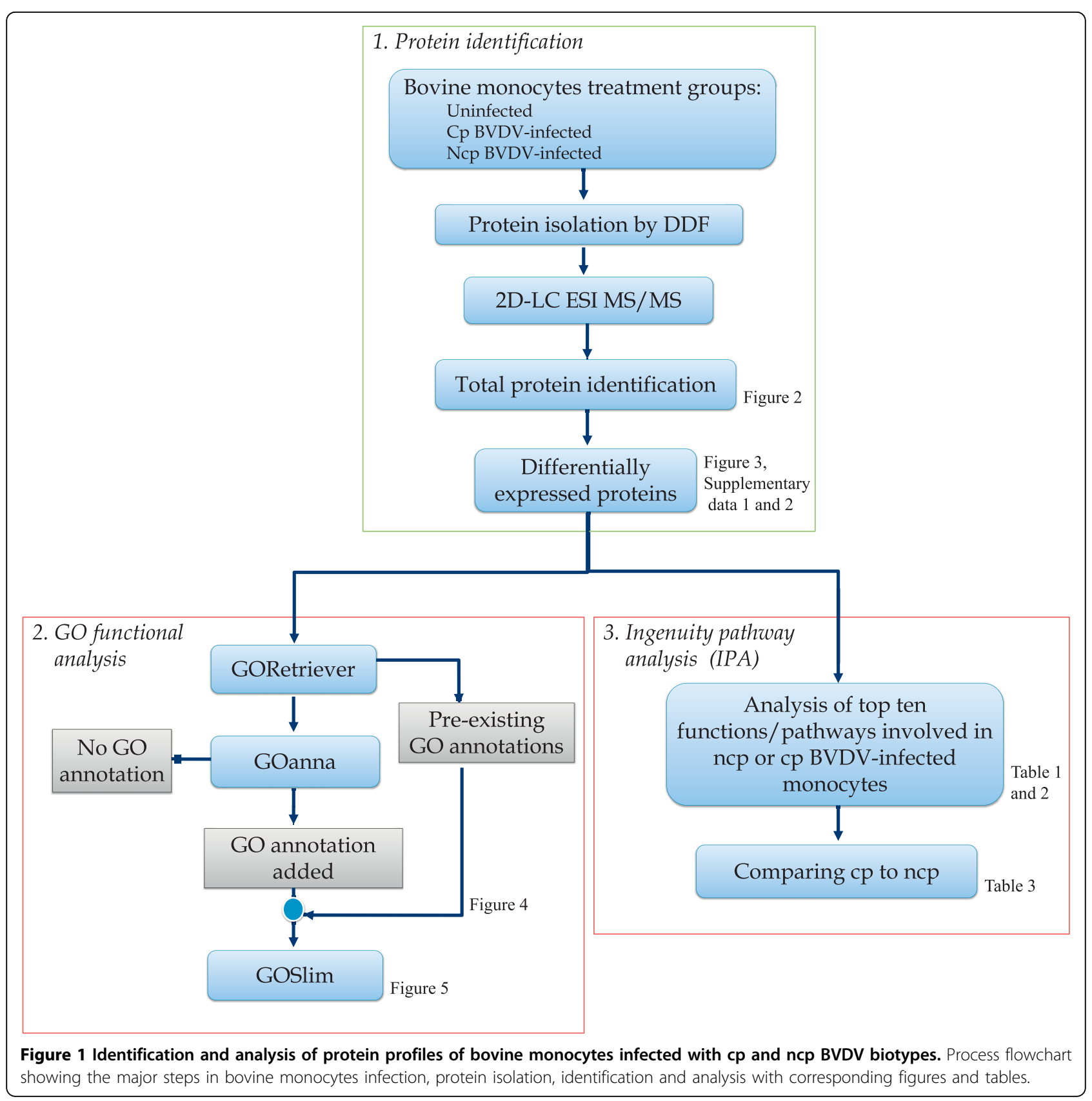

\section{GO Functional analysis of BVDV-infected monocytes}

GO annotations were publicly available for $29.2 \%$ and $22.4 \%$ of the bovine proteins in our ncp and cp BVDV datasets, respectively. We further assigned GO annotations to an additional $62.0 \%$ and $69.3 \%$ of bovine proteins, respectively; bringing the total number of proteins with GO annotation available for functional analysis to 91.2\% and $91.7 \%$ of our ncp and cp BVDV datasets, respectively (Figure 4). This enabled us to perform a comprehensive GO functional modelling. Our GO annotations have been submitted to AgBase, where they will be quality checked and made publicly available. We summarized the GO annotations for bovine proteins differentially expressed in $\mathrm{cp}$ and ncp BVDV infections to identify biological functions in the host response that correspond to infections with these two biotypes. Antioxidant activity, ligand binding, response to stimulus, and extracellular space were over-represented in the ncp BVDV-infected monocytes compared to their cp BVDVinfected counterparts (Figure 5). Transport, enzyme activity, metabolism, and intracellular matters are more highly represented during cp BVDV infection (Figure 5). 


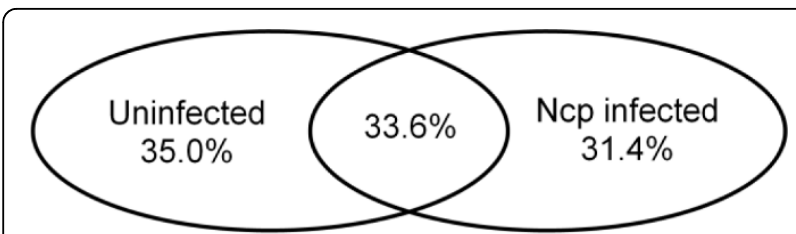

(A)

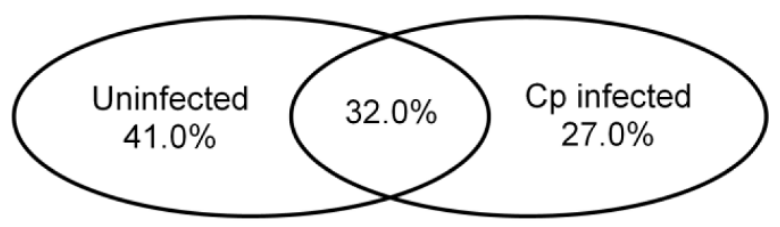

(B)

Figure 2 Distribution of total proteins identified in BVDVinfected monocytes compared to uninfected controls Using 2DLC ESI MS/MS approach a total of 2489, 2356 and 2028 bovine proteins were identified in uninfected, ncp and cp BVDV infected bovine monocytes within two replicates respectively. Distribution of identified proteins compared to uninfected monocytes is shown for (A) ncp infection; and (B) cp infection.

\section{Proteins with significantly altered expression in $\mathrm{cp}$ and} ncp BVDV-infected monocytes: network and pathway analysis

At IPA threshold of significance, 6 and 4 networks and 42 and 33 functions/diseases were significantly represented in the proteomes of ncp and cp BVDV-infected monocytes, respectively. The top ten functions/diseases (ranked based on significance), and the associated

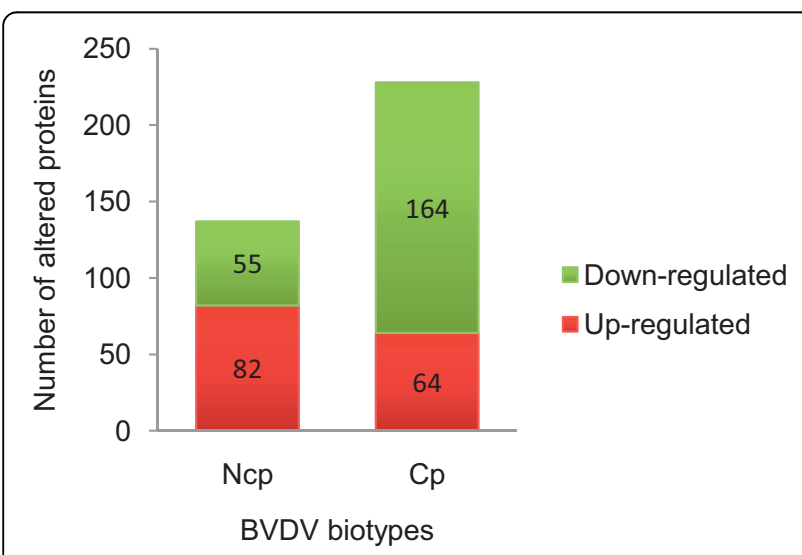

Figure 3 Differentially expressed host proteins in BVDVinfected monocytes compared to uninfected controls

Compared to uninfected monocytes, ncp infection differentially alters the expression of 137 host proteins with $40.2 \%$ downregulated proteins and $59.8 \%$ up-regulated proteins. Whereas, compared to uninfected monocytes, $\mathrm{cp}$ infection alters the expression of 228 host proteins with increasing the percentage of down-regulated proteins to $72 \%$ and decreasing the percentage of up-regulated proteins to $28 \%$ compared to the effect of ncp on uninfected monocytes. signalling pathways are shown in Tables 1 and 2. Analysis of the top ten pathways revealed that pathways representing macropinocytosis signalling, virus entry via endocytic pathway, integrin signalling and primary immunodeficiency signalling were identified only in ncp BVDV-infected monocytes. In contrast, pathways like actin cytoskeleton signalling, RhoA signalling, clathrinmediated endocytosis signalling and interferon signalling were identified only in cp BVDV-infected cells. Of the six common pathways involved in cp and ncp BVDV infection, acute phase response signalling was the most significant for both BVDV biotypes (Tables 1, 2). In each of those six pathways, cp BVDV altered the expression of 33 host proteins compared to the 24 altered proteins due to ncp BVDV infection.

Analysis of the ten most significant IPA functions/diseases for the $\mathrm{cp}$ and ncp biotypes revealed that five were shared, although different proteins were involved in these pathways. The cp BVDV-altered proteins were involved in five cellular-related functions (Tables 1,2). When compared, host proteins differentially expressed in cp and ncp BVDV-infected monocytes included acute phase response signalling, $\mathrm{F} c \gamma$ receptor-mediated phagocytosisin macrophages and monocytes, actin cytoskeleton signalling, antigen presentation pathway, B cell development, RhoA signalling, caveolae-mediated endocytosis signalling, clathrin-mediated endocytosis signalling, IL-10 signalling and interferon signalling (Table 3).

\section{Discussion}

The complex and unique nature of BVDV continues to challenge infectious disease researchers, veterinarians, and the cattle industry. In addition to evading the adaptive immune system, BVDV evade key mechanisms of innate immunity [7]. Although a good understanding of the roles of the two biotypes in the production of persistent infections and the precipitation of mucosal disease has been obtained, there are still unanswered questions regarding the origin of cytopathic viruses and the mechanism by which they cause pathological changes in cells.

In our previous studies we used proteomics to identify host proteins involved in professional antigen presentation altered by cp [9] and protein kinases altered by $\mathrm{cp}$ and ncp [11] BVDV. We have now extended this work by identifications of altered host proteins by ncp and cp BVDV infection based on rigorous statistical methods for peptide identification and control of false positive identifications. Likewise, the workflow for differential protein expression includes multiple testing corrections [12]. Comparing host proteins in $\mathrm{cp}$ and ncp BVDVinfected monocytes to uninfected controls for differential protein expression showed a higher number of affected proteins by cp biotype. In general, cp BVDV 


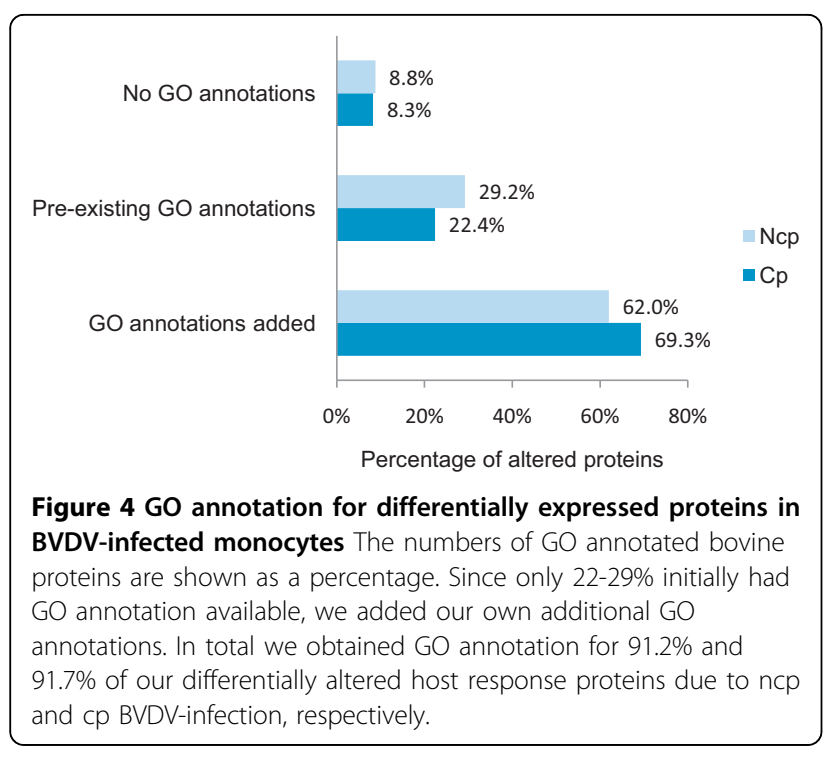

showed more profound effect on the protein expression levels in bovine monocytes with significantly increased number of down-regulated proteins and decreased number of up-regulated proteins compared to the ncp BVDV biotype. This observation is in accord with our previous reports that cp BVDV in general, had more profound effects on antigen uptake mechanisms, TLR, cytokine and co-stimulatory molecule gene and protein kinase protein expression levels in bovine monocytes $[8,9,11]$. The observed significant biotype-related differences might explain the mechanisms by which $\mathrm{cp}$ BVDV, in contrast to ncp biotypes that do not induce cell death, cause pathological changes in infected cells, in particular antigen presenting cells.

In contrast to our previous report on the multiple similarities and some significant biotype-related differences in the monocyte protein expression patterns [11], this new complex modelling approach revealed mostly profound biotype-related differences in all functional groups. This observation strongly supports our hypothesis that low doses of BVDV infection can be crucial to understand the complex pathogenesis of BVDV $[8,9,11]$.

Pathway and network analysis of bovine proteins differentially altered by BVDV also identified significant biotype-related differences. It is known that ncp BVD viruses can establish PI as a result of infection of the embryo early in its development by interfering with a key mechanism of the innate immune system through the interferon (INF) type I production [13]. Since INF is also important in the activation of the adaptive immune response, suppression of this signal may be essential for the establishment of PI [13]. We previously reported that both proteins, CD14 and Mx are increased in

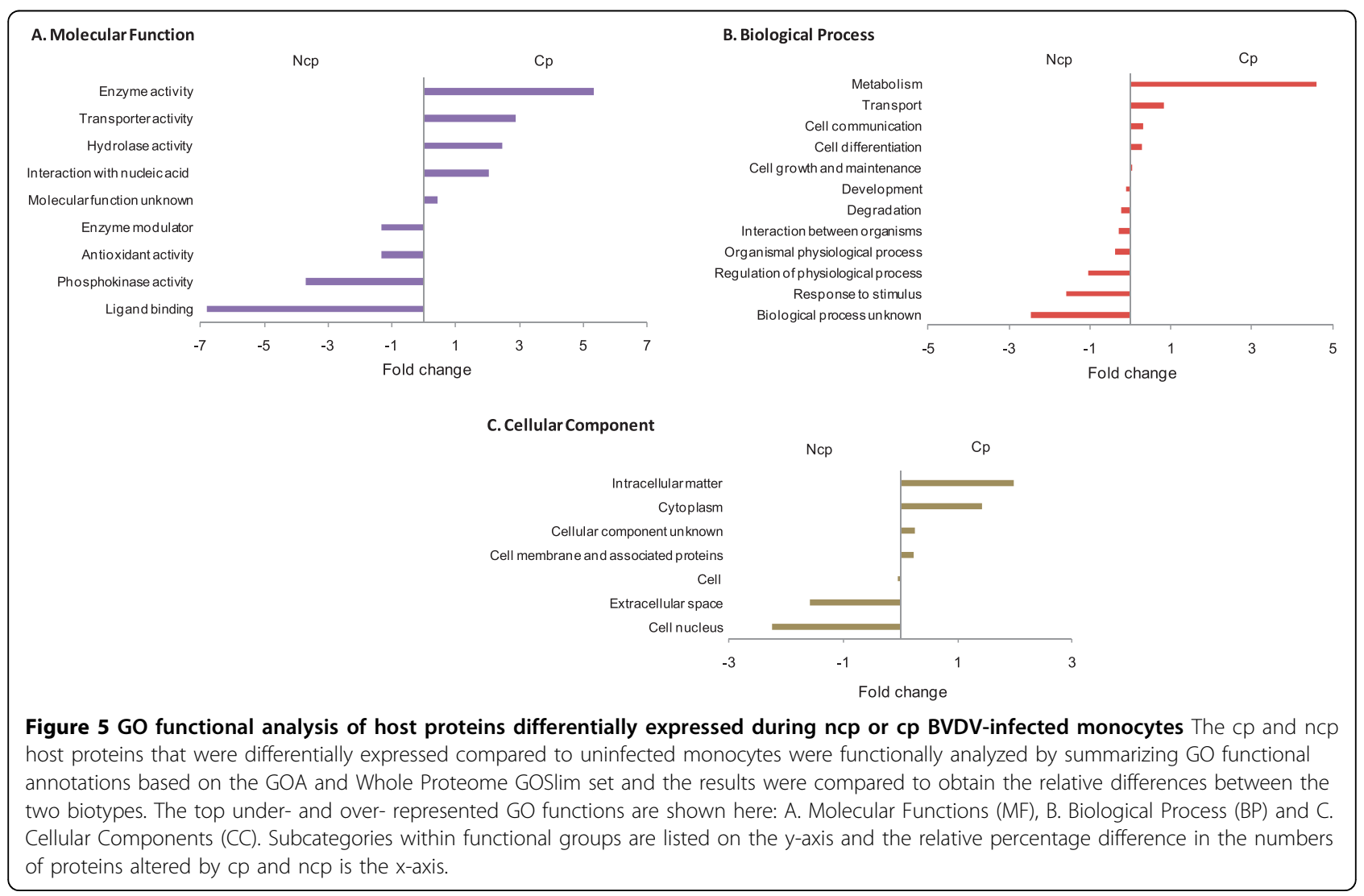




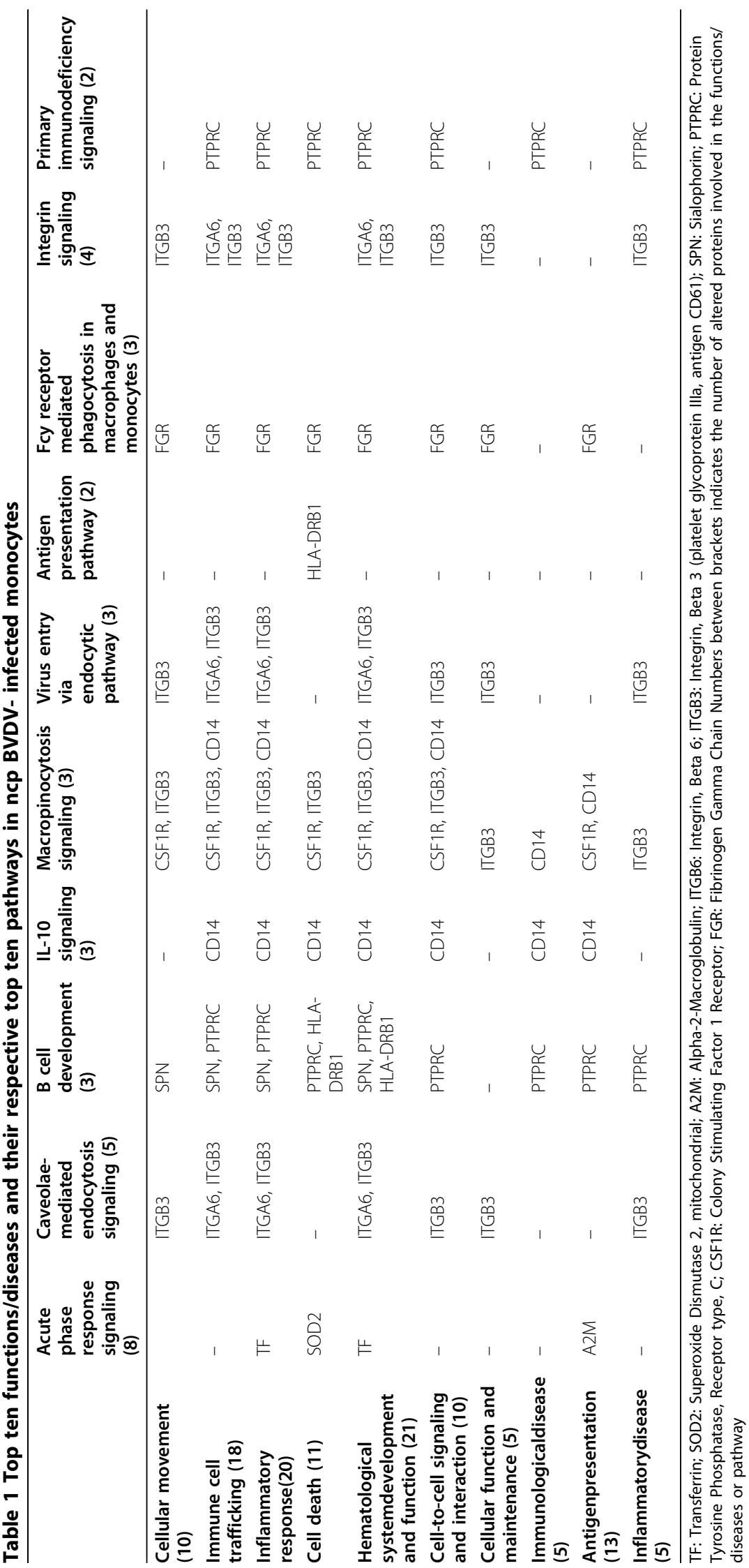




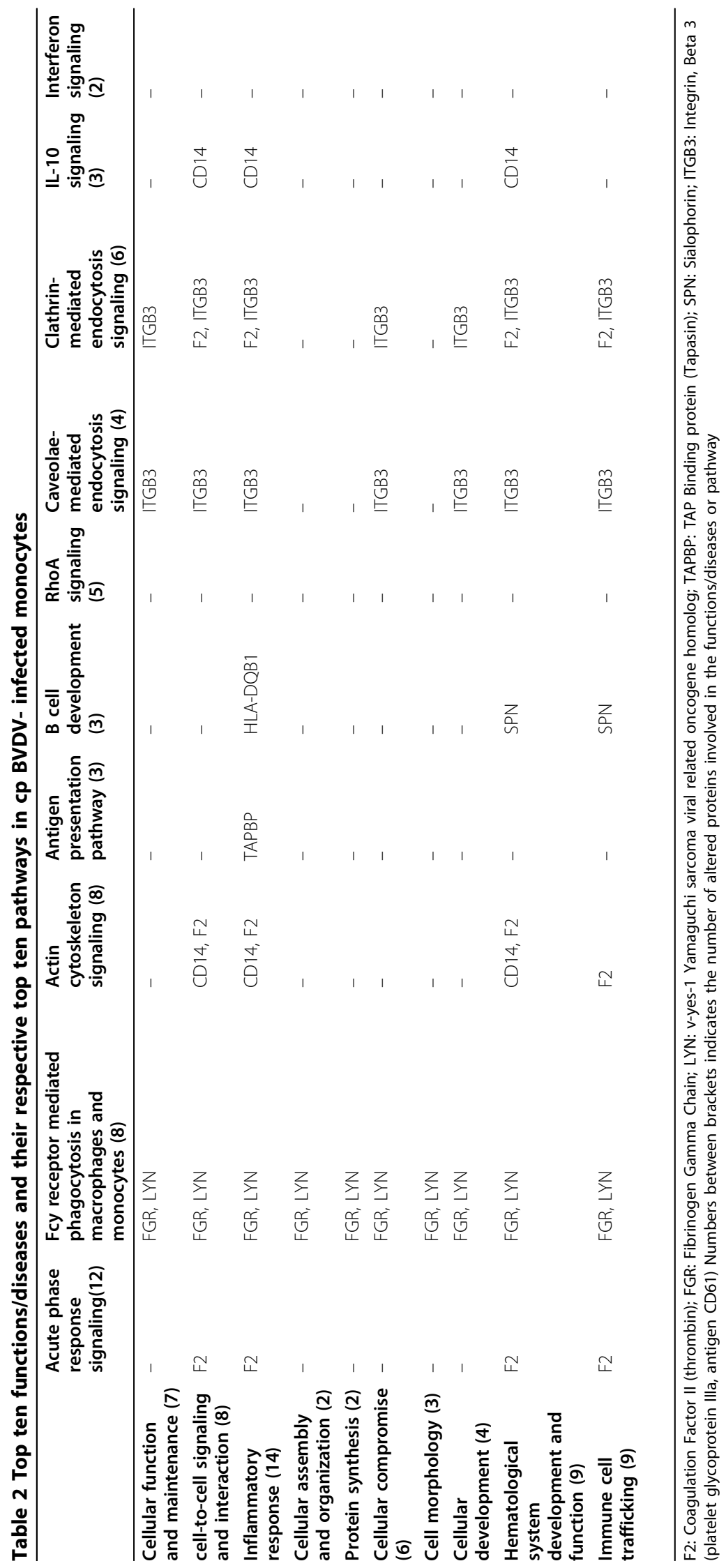


Table 3 Differentially altered proteins represented in top ten immunological pathways when comparing cp to ncp BVDV infection

\begin{tabular}{|c|c|c|c|c|}
\hline \multirow[b]{2}{*}{ Symbol } & \multirow[b]{2}{*}{ Entrez Gene Name } & \multicolumn{2}{|c|}{ Regulation } & \multirow[b]{2}{*}{ Pathways } \\
\hline & & Cp & Ncp & \\
\hline \multirow[t]{2}{*}{ HMOX1 } & heme oxygenase (decycling) 1 & Up & Up & $\begin{array}{l}\text { Acute Phase Response Signaling, Fcy Receptor-mediated Phagocytosis } \\
\text { in }\end{array}$ \\
\hline & & & & Macrophages and Monocytes, IL-10 Signaling \\
\hline ALB & albumin & Down & Down & $\begin{array}{l}\text { Acute Phase Response Signaling, Caveolar-mediated Endocytosis } \\
\text { Signaling }\end{array}$ \\
\hline HP & haptoglobin & Down & Down & Acute Phase Response Signaling \\
\hline SOD2 & superoxide dismutase 2 , mitochondrial & Up & Up & Acute Phase Response Signaling \\
\hline TF & transferrin & Up & Up & $\begin{array}{l}\text { Acute Phase Response Signaling, Clathrin-mediated Endocytosis } \\
\text { Signaling }\end{array}$ \\
\hline $\mathrm{APOH}$ & apolipoprotein H (beta-2-glycoprotein I) & Up & - & Acute Phase Response Signaling \\
\hline APOA1 & apolipoprotein A-I & Up & - & Acute Phase Response Signaling \\
\hline AHSG & alpha-2-HS-glycoprotein & Up & Up & Acute Phase Response Signaling \\
\hline SERPINA1 & $\begin{array}{l}\text { serpin peptidase inhibitor, clade A (alpha-1 } \\
\text { antiproteinase, antitrypsin), member } 1\end{array}$ & Up & Up & Acute Phase Response Signaling \\
\hline $\mathrm{A} 2 \mathrm{M}$ & alpha-2-macroglobulin & Up & Up & Acute Phase Response Signaling \\
\hline FGG & fibrinogen gamma chain & Up & - & Acute Phase Response Signaling \\
\hline $\mathrm{F} 2$ & coagulation factor II (thrombin) & Up & & $\begin{array}{l}\text { Acute Phase Response Signaling, Actin Cytoskeleton Signaling, Clathrin- } \\
\text { mediated Endocytosis Signaling }\end{array}$ \\
\hline \multirow[t]{3}{*}{ ACTR3 } & ARP3 actin-related protein 3 homolog (yeast) & Up & Up & $\begin{array}{l}\text { Fcy Receptor-mediated Phagocytosis in Macrophages and Monocytes, } \\
\text { Actin }\end{array}$ \\
\hline & & & & Cytoskeleton Signaling, RhoA Signaling, Clathrin-mediated Endocytosis \\
\hline & & & & Signaling \\
\hline \multirow[t]{3}{*}{ ARPC2 } & actin related protein $2 / 3$ complex, subunit 2 , & Up & & $\begin{array}{l}\text { Fcy Receptor-mediated Phagocytosis in Macrophages and Monocytes, } \\
\text { Actin }\end{array}$ \\
\hline & $34 \mathrm{kDa}$ & & & Cytoskeleton Signaling, RhoA Signaling, Clathrin-mediated Endocytosis \\
\hline & & & & Signaling \\
\hline \multirow[t]{2}{*}{ EZR } & ezrin & Down & & $\begin{array}{l}\text { Fcy Receptor-mediated Phagocytosis in Macrophages and Monocytes, } \\
\text { Actin }\end{array}$ \\
\hline & & & & Cytoskeleton Signaling, RhoA Signaling \\
\hline LYN & $\begin{array}{l}\text { v-yes-1 Yamaguchi sarcoma viral related } \\
\text { oncogene homolog }\end{array}$ & Up & & Fcy Receptor-mediated Phagocytosis in Macrophages and Monocytes \\
\hline TLN1 & talin 1 & Down & - & Fcy Receptor-mediated Phagocytosis in Macrophages and Monocytes \\
\hline FYB & FYN binding protein (FYB-120/130) & Down & - & Fcy Receptor-mediated Phagocytosis in Macrophages and Monocytes \\
\hline FGR & $\begin{array}{l}\text { Gardner-Rasheed feline sarcoma viral (v-fgr) } \\
\text { oncogene homolog }\end{array}$ & Down & Down & Fcy Receptor-mediated Phagocytosis in Macrophages and Monocytes \\
\hline MYH10 & myosin, heavy chain 10, non-muscle & Up & - & Actin Cytoskeleton Signaling \\
\hline \multirow[t]{2}{*}{ MYL6 } & $\begin{array}{l}\text { myosin, light chain 6, alkali, smooth muscle } \\
\text { and }\end{array}$ & Up & & Actin Cytoskeleton Signaling, RhoA Signaling \\
\hline & non-muscle & & & \\
\hline DIAPH3 & diaphanous homolog 3 (Drosophila) & Down & - & Actin Cytoskeleton Signaling \\
\hline CD14 & CD14 molecule & Up & Up & Actin Cytoskeleton Signaling, IL-10 Signaling \\
\hline IQGAP1 & $\begin{array}{l}\text { IQ motif containing GTPase activating protein } \\
1\end{array}$ & - & Up & Actin Cytoskeleton Signaling \\
\hline HLA-DRB1 & $\begin{array}{l}\text { major histocompatibility complex, class II, DR } \\
\text { beta } 1\end{array}$ & Up & Up & Antigen Presentation Pathway, B Cell Development \\
\hline TAP1 & $\begin{array}{l}\text { transporter 1, ATP-binding cassette, sub-family } \\
\text { B (MDR/TAP) }\end{array}$ & Down & Down & Antigen Presentation Pathway, Interferon Signaling \\
\hline TAPBP & TAP binding protein (tapasin) & Down & - & Antigen Presentation Pathway \\
\hline \multirow[t]{2}{*}{$\begin{array}{l}\text { HLA- } \\
\text { DQB1 }\end{array}$} & major histocompatibility complex, class II, DQ & Down & & B Cell Development \\
\hline & Neta & & & \\
\hline SPN & sialophorin & Down & Down & B Cell Development \\
\hline
\end{tabular}




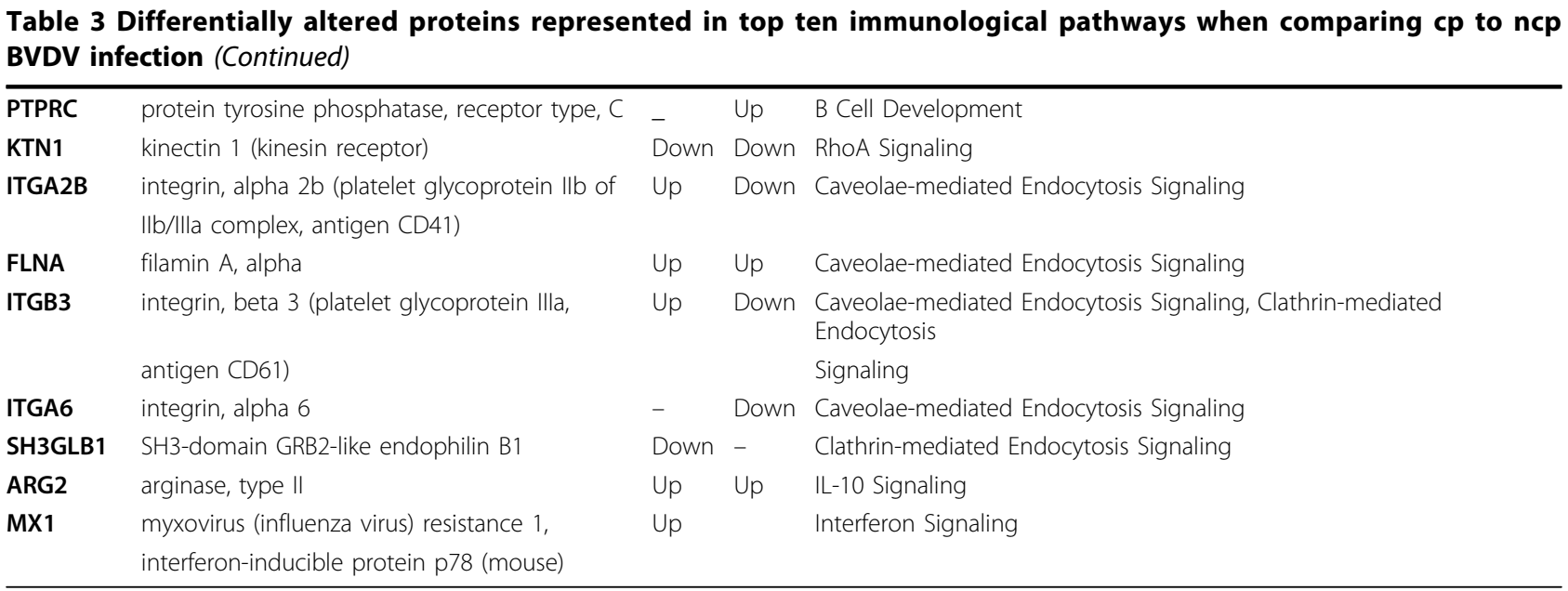

BVDV-infected monocytes. However, in this study that uses stringent protein identification parameters compared to our earlier proteomics methods, expression of Mx significantly increased with cp BVDV infection only. Mx protein is believed to be induced exclusively via signalling through the type I INF receptor $[14,15]$.

The early stages of the host response to infectious agents include a number of physiological changes, collectively known as the acute phase response. Our previous report identified multiple acute phase response proteins altered by cp BVDV [9]. In this study, acute phase pathway was demonstrated to be the first significant pathway in both ncp and cp BVDV infection. Although, ncp and cp viruses altered different numbers of host proteins in general, they had the same effects on the monocyte protein expression levels. The acute phase response is comprised of reactions localized at the site of infection, as well as the initiation of systemic responses, which include a rapid increase in the serum concentration of some proteins, known as acute phase proteins (APP) [16]. Recently, it is becoming clear that viruses interact with iron metabolism. Iron is needed for virus replication, and therefore, by ensuring the infected cell is iron replete, a virus favours its own growth. Moreover, increased concentrations of iron in the body can cause tissue damage and inflammation and affect organ function [17]. For Hepatitis $C$ viral infection, the detrimental effects of excess iron are well documented, and elevated iron status is also associated with increased mortality in HIV-1 infection [17]. Here we show that both cp and ncp BVDV up-regulate transferrin (TF), a negative acute phase protein and a major iron transporter, causing iron overload and exacerbates disease (an animal with an increased serum transferrin level often suffers from iron deficiency anemia). Alternatively, both ncp and cp BVDV down-regulated haptoglobin (HP), a positive acute phase protein capable of binding haemoglobin and removing it from the circulation to prevent iron loss, renal damage and inhibit microbe iron uptake, thus reducing its function as an antioxidant. Although, High HP levels have been reported in the blood of cattle with infections/diseases like mastitis, metritis, traumatic reticulitis, bacterial nephritis and bovine respiratory syncytial virus [16] and many others, there is no literature indicating its involvement in BVDV infection. Therefore, our finding seems to be unexpected, and to investigate the meaning of these two observations, further studies are needed.

Interestingly, among 69 proteins that have been altered by both biotypes only two proteins, integrin alpha 2b (ITGA2B) and integrin beta 3 (ITGB3), were differentially altered by $\mathrm{cp}$ and ncp BVDV biotypes. Integrins are the main cell surface receptors for proteins within the extracellular matrix (ECM); they enable cells to migrate, form strong adhesive junctions, and respond to ECM contact by differentiating and/or proliferating $[18,19]$. Our results indicate that $24 \mathrm{~h}$ ncp BVDV infection decreased the levels of ITGA2B and ITGB3, whereas cp BVDV biotype significantly increased their expression levels. Both integrins are involved in integrin signalling pathway, one of the top ten pathways affected by ncp BVDV-infection. Protein alpha 6 (ITGA6) that was also down-regulated by ncp BVDV, is known to be a member of the integrin family involved in integrin signalling pathway, and was recently shown to be involved in cell differentiation [20]. This finding indicates that ncp BVDV unlike the cp counterpart, inhibited the level of communication of the ECM and cell differentiation. Finally, all the integrins affected by BVDV are also involved in caveolae-mediated endocytosis signalling pathway which was one of the top ten pathways affected by both ncp and cp BVDV biotypes.

In general, the observed effects of cp BVDV in this study are in agreement with our previous reports 
suggesting that $\mathrm{cp}$ BVDV, while promoting the expression of proteins involved in monocyte activation and differentiation, is inhibiting their antigen presentation to immunocompetent $\mathrm{T}$ cells, thus resulting in the uncontrolled inflammation, enhanced viral spread, and impaired anti-viral defense mechanisms in the host. Unlike the cp BVDV biotype, ncp BVDV increased the expression of proteins involved in compensatory survival and inhibition of cell activation mechanisms, promoting virus persistence $[9,11]$.

\section{Conclusions}

In this study, we identified bovine proteins whose expression altered significantly during BVDV infection compared to the uninfected monocytes. Those monocyte protein profiles distinguished between the two biotypes showed that cp BVDV had more profound effect on the protein expression levels with significantly increased number of down-regulated proteins and decreased number of up-regulated proteins compared to the ncp BVDV biotype. The use of GO showed profound biotype-related differences in all GO functional groups, indicating that low doses of BVDV infection can be crucial to understand the complex pathogenesis of BVDV infection. Also, systems biology network modelling identified multiple biotype-related differences in significant biological pathways that could explain the observed biological differences. In particular, our data indicated that only cp BVDV significantly increased the protein expression levels of $\mathrm{Mx}$ protein that is believed to be induced exclusively via signalling through the type I INF receptor. INF receptor signalling activates the adaptive immune responses, and suppression of this signal may be essential for the establishment of persistent infection that could explain the observed biological differences.

In this study, acute phase pathway was demonstrated to be the first significant pathway in both ncp and cp BVDV infection. Although, ncp and cp viruses altered different numbers of proteins in general, they had the same effects on the monocyte protein expression levels. Our finding indicates that ncp BVDV unlike the cp counterpart, inhibited the level of communication of the ECM and cell differentiation thus promoting the establishment of persistent infection. The differences in the expression of the integrins can also mean that cp BVDV infection induces monocytes to differentiate into macrophages, or, alternatively, that monocytes that have already embarked on the differentiation into macrophages, are more susceptible to cp BVDV infection.

Taken together, the combined use of GO information and systems biology network modelling extended our knowledge of the roles of ncp and cp BVDV biotypes in the production of persistent infection and cytopathic effects respectively.

\section{Methods \\ Animals}

Nine conventionally reared, healthy BVDV-free cows from a Holstein herd at the Mississippi State University Dairy Facility were used. The animals have been subjected to a comprehensive vaccination program, including Frontier 4 Plus Vaccine (IBR, BVD, PI3, RSV, Diamond Animal H, Inc). All animal used was approved by The Mississippi State University Institutional Animal Care and Use Committee. Peripheral blood mononuclear cells (PBMC) separated from the animals used in our study were tested for the expression of BVDV E2 transcripts with E2 BVDV specific primers by RT-PCR [8]. As we expected, all animals were BVDV mRNA-free (data not shown).

\section{Cell preparation}

Blood samples $(150 \mathrm{ml})$ were collected into Blood Collection Tubes $(16 \times 100 \mathrm{~mm}$, Tyco Healthcare) by jugular venipuncture. Bovine PBMC were separated as described elsewhere [8,21]. Briefly, PBMC were isolated using Histopaque gradients $(1.077 \mathrm{~g} / \mathrm{ml}$, Amersham Biosciences) and resuspended in RPMI-1640 supplemented with $10 \%$ FBS, 1\% Glutamax-1 (Invitrogen), 5×10- 5 M 2-mercaptoethanol and $100 \mathrm{IU} / \mathrm{ml}$ Gentamicin (Invitrogen). Monocytes were separated from PBMC as described elsewhere [22]. Briefly, $40 \mathrm{ml}$ of PBMC suspension $(5 \times 108$ cells $)$ was added to Petri-dish $(150 \times 25 \mathrm{~mm}, \mathrm{BD}$ sciences) for $2 \mathrm{~h}$ at $37^{\circ} \mathrm{C}$. Non-adherent cells were removed and the adherent cells were washed twice in PBS (Invitrogen). The yield of adherent cells was 20$30 \%$ of total PBMC number. After removing non-adherent populations (mostly $\mathrm{T}$ and $\mathrm{B}$ cells), adherent cells were incubated with mAbs to CD14 (MM61A, VMRD) followed by the addition of magnetic beads conjugated with mouse anti-IgG1 (Miltenyi Biotech, Auburn, CA) [21]. CD14+ monocytes were positively selected by using magnetic cell separation technique according to the manufacturer's instructions (Miltenyi Biotech). The final yield of bovine monocytes was $2-3 \%$ of total PBMC number.

\section{BVDV stocks and infection}

BVDV biotypes were prepared as described elsewhere [12]. Briefly, the NADL (cp) biotype of BVDV was obtained from the American Type Culture Collection (ATCC) and amplified by growth in the bovine turbinate (BT) cell line (ATCC) according to the manufacturer's handling procedures. For infection of BT cells, virus dilutions were made in DMEM with $4 \mathrm{mM} \mathrm{L}$-glutamine, 
$4.5 \mathrm{~g} / \mathrm{l}$ glucose, $1.5 \mathrm{~g} / \mathrm{l}$ sodium bicarbonate and $10 \%$ horse serum. To measure the infectivity of the NADL biotype, the quantal method of Reed and Muench was performed and the tissue culture infectious dose 50 (TCID50) was determined. For the ncp BVDV biotype NY, we used the TCID50 suggested by the manufacturer (ATCC). To select the dose of cp BVDV that did not have a cytopathic effect on monocytes cultured for $48 \mathrm{~h}$ we assessed the viability of the infected cells by using trypan blue and light microscopy. BVDV biotype NADL at the multiplicity of infection (MOI) 0.002 had not affected the viability of bovine monocytes after $48 \mathrm{~h}$ of infection (data not shown). $5 \times 106$ monocytes were added to each well of a 6 well tissue culture plate and infected with cp and ncp BVDV biotypes at the same MOI of 0.002 for $24 \mathrm{~h}$. After infection, at least 107 cells were pooled in one tube. All data were determined using triplicate monocyte cultures.

\section{Protein extraction by DDF}

Differential detergent fractionation (DDF) sequentially extracts proteins using a series of detergents with increasing ionic strength, leading to an increase in proteome coverage. Proteins were isolated using DDF as previously described [23,24]. Briefly, cytosolic proteins were isolated and depleted by repeated washes in digitonin buffer. After the digitonin washes, proteins were sequentially extracted using triton X-100 (TX), deoxycholate (DOC), tween 40, and SDS buffers, respectively. To evaluate the quality of isolated proteins, $1 \%$ of the protein samples were compared using 10\% SDS-PAGE (data not shown). For each of the detergent fractions, equal amounts of protein were precipitated with $25 \%$ trichloroacetic acid to remove salts and detergents. Protein pellets were solubilized and then digested with 100 ng of trypsin (50:1 ratio of substrate to enzyme) overnight at $37^{\circ} \mathrm{C}$. Peptides were desalted using a peptide microtrap (Michrom BioResources, Inc.) and eluted by a $0.1 \%$ trifluoroacetic acid, $95 \%$ acetonitrile solution. Desalted peptides were dried and resuspended in $0.1 \%$ formic acid.

\section{D-LC ESI MS ${ }^{2}$}

Proteomic analysis was carried out with duplicate samples of untreated, cp and ncp-BVDV infected bovine monocytes using 2D-LC ESI MS ${ }^{2}$ as described elsewhere $[23,24]$. Briefly, LC analysis was accomplished by strong cation exchange (SCX) followed by reverse phase (RP) liquid chromatography (LC) coupled directly in line with electrospray (ESI) ion trap MS. Each DDF fraction samples from three different infections were loaded into a LC gradient ion exchange system including a Thermo Separations P4000 quaternary gradient pump (ThermoElectron Corporation) coupled with a $0.32 \times 100 \mathrm{~mm}$
BioBasic SCX column and run three times. A flow rate of $3 \mu \mathrm{l} /$ minwas used for both SCX and RP columns. A salt gradient was applied in steps of $0,5,10,15,20,25$, $30,35,40,45,50,57,64,71,79,90,110,300$, and 700 $\mathrm{mM}$ ammonium acetate in $5 \%$ acetonitrile, $0.1 \%$ formic acid and the resultant peptides were loaded directly into the sample loop of a $0.18 \times 100 \mathrm{~mm}$ BioBasic C18 RP LC column of a Proteome X workstation (ThermoElectron). The RP gradient used $0.1 \%$ formic acid in acetonitrile and increased the acetonitrile concentration in a linear gradient from $5 \%$ to $30 \%$ in $30 \mathrm{~min}$ and then $30 \%$ to $65 \%$ in 9 min followed by $95 \%$ for $5 \mathrm{~min}$ and $5 \%$ for $15 \mathrm{~min}$.

The spectrum collection time was 59 min for every SCX step. The LCQ Deca ion trap mass spectrometer (ThermoElectron) was configured to optimize the duty cycle length with the quality of data acquired by alternating between a single full MS scan followed by three tandem MS scans on the three most intense precursor masses from full scan. The collision energy was normalized to $35 \%$. Dynamic mass exclusion windows were set at $2 \mathrm{~min}$, and all of the spectra were measured with an overall mass/charge $(\mathrm{m} / \mathrm{z})$ ration range of $200-2000$.

\section{Protein identification and differential protein expression}

Proteins were identified and analyzed as previously described [12]. All searches were done using TurboSEQUEST $^{\mathrm{mm}}$ (Bioworks Browser 3.2; ThermoElectron) [25]. Mass spectra and tandem mass spectra were searched against an in silico trypsin-digested non-redundant protein database of Bos taurus downloaded from National Center for Biotechnology Institute (NCBI). Cysteine carboxyamidomethylation and methionine single and double oxidation were included in the search criteria. Decoy searches from a randomized version of the bovine protein database were conducted with tandem mass spectra as described above. The probability for peptide identification was estimated using a method described for Sequest data analysis and was set at $\mathrm{p}<0.05$ [26]. Probabilities of protein identifications being incorrect were calculated using published methods $[27,28]$. Differential protein expression analysis based on $\Sigma$ Xcorr was carried out as described in ProtQuant [29]. To correct for multiple testing, we determined the false discovery rate (FDR) for $\mathrm{p}$ value using published methods [30].

\section{Gene Ontology Annotation}

Gene ontology (GO) analysis was carried using AgBase tools [31] to identify the molecular functions, biological processes and cellular component represented in our protein datasets. GORetriver tool was used to obtain all pre-existing GO annotations available for known proteins in our datasets. In addition, we used GOanna to provide additional GO annotation (i.e. predicted based 
on sequence orthologes and analysis of functional domains) for bovine proteins without existing annotation. All GO annotations for our datasets were grouped into more generalized categories using GOSlimViewer and summarized using the GOA and Whole Proteome GOSlim set. Subcategories in each of the three GOSlim functional categories are shown as a fold change between the percentages of GO terms identified in cp to those of ncp BVDV-infected monocytes in Figure 5.

\section{Modelling using Ingenuity Pathway Analysis}

To visualize and explore networks which are significantly represented in our proteomic datasets we used Ingenuity Pathways Analysis (IPA; Ingenuity system, California). Only differentially expressed host proteins in BVDV-infected monocytes were analyzed by IPA. Each gene identifier was mapped to its corresponding gene object in the Ingenuity Pathways Knowledge Base (IPKB). IPKB selects "focus genes" to be used for generating biological networks and computes a score for each network from P-value which indicates the likelihood of the focus genes in a network being found together due to chance. We selected networks scoring $\geq 2$, which have $>99 \%$ confidence of not being generated by chance $[32,33]$. Biological functions are assigned to each network by using annotations from scientific literature and stored in the IPKB. Fisher exact test is used to calculate the P-value determining the probability of each biological function/disease or pathway being assigned by chance. We used immunological cells as a filter and a $P$-value $\leq 0.05$ to select highly significant functions/disease and pathways represented in our proteomic datasets.

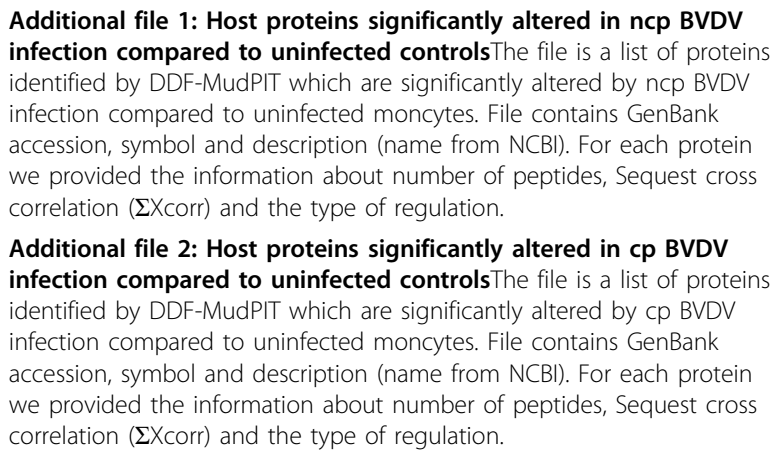

Additional file 2: Host proteins significantly altered in cp BVDV infection compared to uninfected controlsThe file is a list of proteins identified by DDF-MudPIT which are significantly altered by cp BVDV infection compared to uninfected moncytes. File contains GenBank accession, symbol and description (name from NCBI). For each protein we provided the information about number of peptides, Sequest cross correlation ( $\Sigma$ Xcorr) and the type of regulation.

\section{Acknowledgements}

The authors wish to acknowledge Ken Pendarvis and the MSU LSBI facility for performing mass spectrometry and protein analysis of bovine monocyte DDF.

This article has been published as part of BMC Bioinformatics Volume 11 Supplement 6, 2010: Proceedings of the Seventh Annual MCBIOS Conference. Bioinformatics: Systems, Biology, Informatics and Computation.
The full contents of the supplement are available online at http://www.biomedcentral.com/1471-2105/11?issue=S6.

\section{Author details}

'Department of Basic Sciences, Mississippi State University, Mississippi State MS 39762, USA. ${ }^{2}$ Institute of Digital Biology, Mississippi State University, Mississippi State, MS 39762, USA

\section{Authors' contributions}

MA performed the data generation, analyzed and interpreted proteomic data and wrote the draft of the manuscript. FM, BN and LP helped to draft the manuscript. FM co-ordinated the project and assisted with functional analysis. BN participated in analyzing the proteomics data and assisted with system biology modelling. LP initiated the project and helped in interpretation of data. Study was part of MA graduate work. LP is the corresponding author and the major professor of MA. All authors read and approved the final manuscript.

\section{Competing interests}

The authors declare that they have no competing interests.

Published: 7 October 2010

\section{References}

1. Kobrak A, Weber EL: [Bovine diarrhea virus: an update]. Rev Argent Microbiol 1997, 29(1):47-61.

2. Houe $\mathrm{H}$ : Epidemiological features and economical importance of bovine virus diarrhoea virus (BVDV) infections. Vet Microbiol 1999, 64(2-3):89-107.

3. Hamers C, Dehan P, Couvreur B, Letellier C, Kerkhofs P, Pastoret PP: Diversity among bovine pestiviruses. Vet J 2001, 161(2):112-122.

4. Fulton RW, Ridpath JF, Ore S, Confer AW, Saliki JT, Burge LJ, Payton ME: Bovine viral diarrhoea virus (BVDV) subgenotypes in diagnostic laboratory accessions: distribution of BVDV1a, 1b, and 2a subgenotypes. Vet Microbiol 2005, 111(1-2):35-40.

5. Brock KV: The persistence of bovine viral diarrhea virus. Biologicals 2003, 31(2):133-135.

6. Brownlie J, Clarke MC, Howard CJ, Pocock DH: Pathogenesis and epidemiology of bovine virus diarrhoea virus infection of cattle. Ann Rech Vet 1987, 18(2):157-166.

7. Brock KV: The many faces of bovine viral diarrhea virus. Vet Clin North Am Food Anim Pract 2004, 20(1):1-3.

8. Boyd BL, Lee TM, Kruger EF, Pinchuk LM: Cytopathic and non-cytopathic bovine viral diarrhoea virus biotypes affect fluid phase uptake and mannose receptor-mediated endocytosis in bovine monocytes. Vet Immunol Immunopathol 2004, 102(1-2):53-65.

9. Lee SR, Nanduri B, Pharr GT, Stokes JV, Pinchuk LM: Bovine viral diarrhea virus infection affects the expression of proteins related to professional antigen presentation in bovine monocytes. Biochim Biophys Acta 2009, 1794(1):14-22

10. Franchini M, Schweizer M, Matzener P, Magkouras I, Sauter KS, Mirkovitch J, Peterhans $E$, Jungi TW: Evidence for dissociation of TLR mRNA expression and TLR agonist-mediated functions in bovine macrophages. Vet Immunol Immunopathol 2006, 110(1-2):37-49.

11. Pinchuk GV, Lee SR, Nanduri B, Honsinger KL, Stokes JV, Pinchuk LM: Bovine viral diarrhea viruses differentially alter the expression of the protein kinases and related proteins affecting the development of infection and anti-viral mechanisms in bovine monocytes. Biochim Biophys Acta 2008, 1784(9):1234-1247.

12. Pendarvis K, Kumar R, Burgess SC, Nanduri B: An automated proteomic data analysis workflow for mass spectrometry. BMC Bioinformatics 2009, 10(Suppl 11):S17.

13. Peterhans $E$, Jungi TW, Schweizer M: BVDV and innate immunity. Biologicals 2003, 31(2):107-112.

14. von Wussow $P$, Jakschies $D$, Hochkeppel HK, Fibich $C$, Penner L, Deicher $H$ : The human intracellular Mx-homologous protein is specifically induced by type I interferons. Eur J Immunol 1990, 20(9):2015-2019.

15. Baigent SJ, Zhang G, Fray MD, Flick-Smith H, Goodbourn S, McCauley JW: Inhibition of beta interferon transcription by noncytopathogenic bovine viral diarrhea virus is through an interferon regulatory factor 3dependent mechanism. J Virol 2002, 76(18):8979-8988. 
16. Heegaard PM, Godson DL, Toussaint MJ, Tjornehoj K, Larsen LE, Viuff B, Ronsholt $L$ : The acute phase response of haptoglobin and serum amyloid A (SAA) in cattle undergoing experimental infection with bovine respiratory syncytial virus. Vet Immunol Immunopathol 2000, 77(12):151-159.

17. Drakesmith $H$, Prentice $A$ : Viral infection and iron metabolism. Nat Rev Microbiol 2008, 6(7):541-552.

18. Delon I, Brown NH: Integrins and the actin cytoskeleton. Curr Opin Cell Biol 2007, 19(1):43-50.

19. Le Clainche C, Carlier MF: Regulation of actin assembly associated with protrusion and adhesion in cell migration. Physiol Rev 2008, 88(2):489-513.

20. Meighan CM, Schwarzbauer JE: Temporal and spatial regulation of integrins during development. Curr Opin Cell Biol 2008, 20(5):520-524.

21. Lee SR, Pharr GT, Boyd BL, Pinchuk LM: Bovine viral diarrhea viruses modulate toll-like receptors, cytokines and co-stimulatory molecules genes expression in bovine peripheral blood monocytes. Comp Immunol Microbiol Infect Dis 2008, 31(5):403-418.

22. Kruger EF, Boyd BL, Pinchuk LM: Bovine monocytes induce immunoglobulin production in peripheral blood B lymphocytes. Dev Comp Immunol 2003, 27(10):889-897.

23. Lee SR, Pharr GT, Cooksey AM, McCarthy FM, Boyd BL, Pinchuk LM: Differential detergent fractionation for non-electrophoretic bovine peripheral blood monocyte proteomics reveals proteins involved in professional antigen presentation. Dev Comp Immunol 2006, 30(11):1070-1083.

24. McCarthy FM, Burgess SC, van den Berg BH, Koter MD, Pharr GT: Differential detergent fractionation for non-electrophoretic eukaryote cell proteomics. J Proteome Res 2005, 4(2):316-324.

25. Eng JK, McCormack AL, Yates JR: An approach to correlate tandem mass spectral data of peptides with amino acid sequences in a protein database. J Am Soc Mass Spectrom 1994, 5:976-989.

26. Qian WJ, Liu T, Monroe ME, Strittmatter EF, Jacobs JM, Kangas LJ, Petritis K, Camp DG, Smith RD 2nd: Probability-based evaluation of peptide and protein identifications from tandem mass spectrometry and SEQUEST analysis: the human proteome. J Proteome Res 2005, 4(1):53-62.

27. Lopez-Ferrer D, Martinez-Bartolome S, Villar M, Campillos M, MartinMaroto F, Vazquez J: Statistical model for large-scale peptide identification in databases from tandem mass spectra using SEQUEST. Anal Chem 2004, 76(23):6853-6860.

28. MacCoss MJ, Wu CC, Yates JR 3rd: Probability-based validation of protein identifications using a modified SEQUEST algorithm. Anal Chem 2002, 74(21):5593-5599.

29. Bridges SM, Magee GB, Wang N, Williams WP, Burgess SC, Nanduri B: ProtQuant: a tool for the label-free quantification of MudPIT proteomics data. BMC Bioinformatics 2007, 8(Suppl 7):S24.

30. Benjamini $Y$, Hochberg $Y$ : Controlling the false discovery rate: a practical and powerful approach to multiple testing. Journal of the Royal Statistical Society: Series B (Statistical Methodology) 1995, 57:289-300.

31. McCarthy FM, Bridges SM, Wang N, Magee GB, Williams WP, Luthe DS, Burgess SC: AgBase: a unified resource for functional analysis in agriculture. Nucleic Acids Res 2007, 35(Database issue):D599-603.

32. Gerling IC, Singh S, Lenchik NI, Marshall DR, Wu J: New data analysis and mining approaches identify unique proteome and transcriptome markers of susceptibility to autoimmune diabetes. Mol Cell Proteomics 2006, 5(2):293-305.

33. Peddinti D, Nanduri B, Kaya A, Feugang JM, Burgess SC, Memili E: Comprehensive proteomic analysis of bovine spermatozoa of varying fertility rates and identification of biomarkers associated with fertility. BMC Syst Biol 2008, 2:19.

doi:10.1186/1471-2105-11-S6-S9

Cite this article as: Ammari et al.: Analysis of Bovine Viral Diarrhea Viruses-infected monocytes: identification of cytopathic and noncytopathic biotype differences. BMC Bioinformatics 2010 11(Suppl 6):S9.

\section{Submit your next manuscript to BioMed Central and take full advantage of:}

- Convenient online submission

- Thorough peer review

- No space constraints or color figure charges

- Immediate publication on acceptance

- Inclusion in PubMed, CAS, Scopus and Google Scholar

- Research which is freely available for redistribution

Submit your manuscript at www.biomedcentral.com/submit
Biomed Central 